\title{
ANALYSIS OF THE OECD/NEA SFR BENCHMARK WITH ANTS REDUCED-ORDER NODAL DIFFUSION SOLVER AND THE SERPENT MONTE CARLO CODE
}

\author{
Marton Szogradi \\ VTT Technical Research Centre of Finland Ltd \\ P.O. Box 1000, FI-02044 VTT, Finland \\ marton.szogradi@vtt.fi
}

\begin{abstract}
In order to meet modern industrial and scientific demands the Kraken multi-physics platform's development was recently launched at VTT Technical Research Centre of Finland. The neutronic solver of the framework consists of two calculation chains, providing full core solutions by the Serpent high fidelity code (1) and the AFEN/FENM-based reduced-order diffusion solver called Ants (2) capable of handling square and hexagonal geometries in steady-state. Present work introduces the simulation of a large 3600 MWth Sodium-cooled Fast Reactor (SFR) described within the activities of the Working Party on Scientific Issues of Reactor Systems (WPRS) of OECD. Full-core 3D results were obtained by Serpent for carbide- and oxide-fuel cores, moreover group constants were generated for Ants utilizing 2D super-cell and single assembly infinite lattice models of Serpent. The continuous-energy Monte Carlo method provided the reference results for the verification of the reduced-order method. Implementing the spatially homogenized properties, 3D solutions were obtained by Ants as well for both core configurations. Comparison was made between the various core designs and codes based on reactivity feedbacks (Doppler constant, sodium voiding, control rod worth) considering power distributions. Regarding reactivity sensitivity on geometry, axial fuel- and radial core expansion coefficients were evaluated as well.
\end{abstract}

KEYWORDS: SFR, nodal diffusion solver, Ants, Serpent

\section{INTRODUCTION}

Benchmark data from report [1] was used to construct 3D full-core SFR models in Serpent with respect to given boundary conditions. Two configurations, one of carbide (CA) and one of oxide $(\mathrm{OX})$ fuel was defined with Beginning of Cycle $(\mathrm{BoC})$ isotopic composition. In Section 2 the core design is discussed and the performance of the models under various perturbations e.g. Doppler effect, sodium voiding and diagrid expansion. In Section 3 few-group constants' generation is described with 2D spatial homogenization highlighting the sensitivity of various condensation methods. In Section 4 the diffusion solver is introduced briefly regarding theoretical background and capabilities. Finally the assessment of Ants will be carried out with respect to 3D Serpent results. 


\section{SERPENT FULL-CORE 3D MODELS}

Both studied cores have medium power density, structural materials are identical (e.g. Oxide Strenghtened Steel - ODS cladding) and helium bonded fuel pellets. Each design bears benefits and disadvantages therefore considering both configurations gives a wider perspective regarding code versatility and reliability. The CA core's main motivation was to achieve a low linear fuel temperature gradient providing an increased safety margin for pellet-centerline temperature. The OX configuration was conceived with the "fat pin with small wire" approach i.e. the core could reach self-breeding without fertile blanket. The models represent BoC conditions thus the helium bonded fuel pellets are not in contact with the cladding.

\subsection{Core Configurations}

The CA core contains 487 fuel assemblies (206 inner / 201 outer), 270 radial reflector elements and 27 control assemblies whereas 18 and 9 assemblies represent the primary and secondary control system, respectively. The $(\mathrm{U}, \mathrm{Pu}) \mathrm{C}$ pellets are whole, the fuel cladding is made of ODS alloy while the shroud tube and the axial reflector elements consist of EM10 SS (Euralloy). The OX core has 453 fuel assemblies (225 inner / 228 outer) and the same radial reflector shield and control systems as the $\mathrm{CA}$. The $(\mathrm{U}, \mathrm{Pu}) \mathrm{O}_{2}$ pellets are hollow with ODS cladding and similar EM10 structures as the CA fuel. Major geometry and material properties of the cores are tabulated in Table 1. The core layouts are shown in Fig. 1. Regarding the control rods, both system contains $\mathrm{B}_{4} \mathrm{C}$ pellets as absorber material, whereas the primary rods have natural isotopic composition while the secondary assemblies are loaded with enriched $\left(90 \%{ }^{10} \mathrm{~B}\right)$ boron carbide.

Table 1: Comparison of Core Design.

\begin{tabular}{|c|c|c|}
\hline Property & CA & OX \\
\hline Overall height $(\mathrm{cm})$ & 301.70 & 311.16 \\
\hline Active core height $(\mathrm{cm})$ & 100.56 & 100.56 \\
\hline Subassembly pitch $(\mathrm{cm})$ & 20.9889 & 21.2205 \\
\hline Number of fuel pins (-) & 469 & 271 \\
\hline Pellet radius (mm) & 3.319 & 4.742 \\
\hline Inner/Outer core Pu content $(\mathrm{wt} \%)$ & $13.76 / 17.60$ & $15.81 / 17.60$ \\
\hline
\end{tabular}

\subsection{Present Models}

Full-core 3D model inputs were built for Serpent 2.1.31 [2] based on Ref. [1] whereas He pressure (atomic density) was chosen to be 10 bar according to MOX fuel recommendations reported by the Halden Reactor Project [3]. The fuel pins of the inner and outer core were divided into 5 axial nodes representing different $\mathrm{Pu}$ enrichments. The control assemblies were fully withdrawn from the core in every test case except for the control rod worth (CRW) simulations. A total of 1700 cycles (1500 active / 200 inactive cycles) were calculated with 600000 neutron histories/cycle 


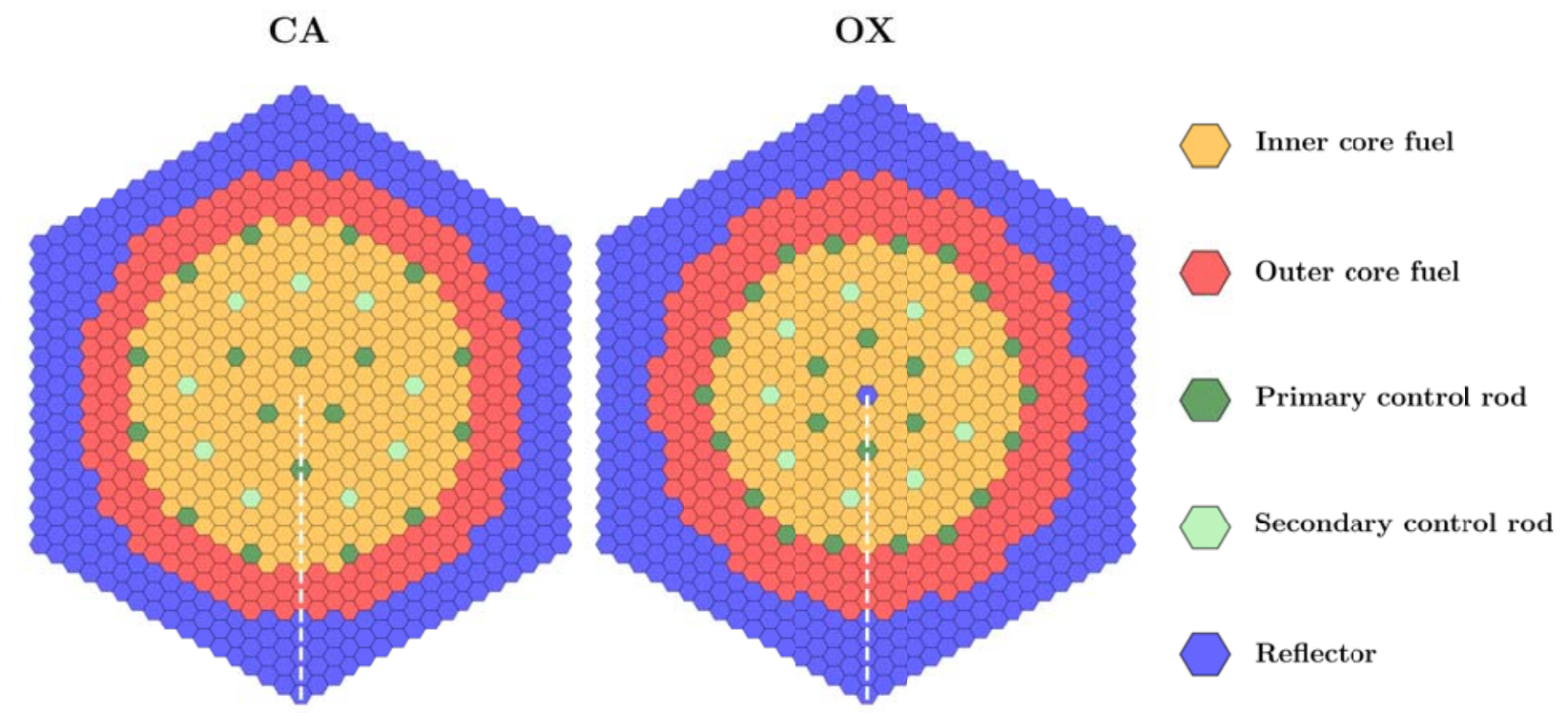

Figure 1: SFR Core Layouts.

utilizing the JEFF-3.1.2 library. Besides the benchmark criticality case Doppler constants $\left(K_{\mathrm{D}}\right)$ were calculated with $1000 \mathrm{~K}$ fuel temperature raise ${ }^{*}\left(\Delta T_{\mathrm{f}}\right)$ for each configuration:

$$
K_{\mathrm{D}}=T_{\mathrm{f}} \frac{d \rho}{d T} \approx \frac{\rho_{\mathrm{l}}-\rho_{0}}{\ln \left(T_{\mathrm{f}, 1} / T_{\mathrm{f}, 0}\right)}(\mathrm{pcm})
$$

where index 0 refers to the benchmark case, index 1 denotes the perturbed case and $\rho$ is reactivity. The sodium void reactivity (SVR) and CRW values were obtained by simply taking the difference between the nominal and the perturbed cases as $\mathrm{SVR}=\rho_{0}-\rho_{\mathrm{SV}, 100}$ and $\mathrm{CRW}=\rho_{0}-\rho_{\mathrm{CRs} \text {,in }}$, respectively. The axial fuel and radial core expansion reactivity feedback was calculated with the same logic as SVR and CRW comparing the two cases whereas the $L_{\mathrm{f}}$ length of the fuel slug had to be modified in the Serpent model by:

$$
L_{\mathrm{f}}\left(T_{1}\right)=L_{\mathrm{f}}\left(T_{0}\right)\left[1+\alpha_{\mathrm{CA} / \mathrm{OX}}\left(T_{1}-T_{0}\right)\right](\mathrm{cm}),
$$

yielding a $\Delta L_{\mathrm{f}} / L_{\mathrm{f}}$ of $+1.29 / 1.77 \%$ for $\mathrm{CA} / \mathrm{OX}$ with corresponding fuel density reduction, where $\alpha_{\mathrm{CA} / \mathrm{OX}}$ is the linear expansion coefficient of the fuel [4,5]. Radial expansion of core diagrid was derived from the pitch $(p)$ perturbation:

$$
p_{1}\left(T_{1}\right)=p_{0}\left(T_{0}\right)\left[1+\alpha_{\mathrm{rad}, \mathrm{g}}\left(T_{1}-T_{0}\right)\right](\mathrm{m}),
$$

giving a $\Delta p / p$ of $+0.99 \%$ for both cases based on the same 316L SS structural material, where $\alpha_{\text {rad,g }}$ was the global linear expansion coefficient of the diagrid.

${ }^{*}$ The benchmark suggested doubling the fuel temperature for both CA/OX cores however admittedly such temperatures were not physical. In order to approximate these temperatures as close as possible and keep temperatures below fuel melting point the $1000 \mathrm{~K}$ increase was applied. 


\subsection{Serpent Results}

Results were compared with benchmark data summarized in [6] and studies conducted by Nikitin et al. $[7,8]$ with Serpent. The benchmark averages are distorted by codes with homogeneous cell description while $[7,8]$ represent a heterogeneous approach. One has to account for inherent differences of code versions and boundary conditions e.g. cross section libraries, Table 2 summarizes the results. In general the discrepancies are acceptable considering the $\pm 500 \mathrm{pcm}$ uncertainty ranges in [6] however the SVR values highlight larger differences. Axial fuel and radial diagrid expansion feedback indicated that the cores are more sensitive to pitch perturbation than thermal elongation of the fuel slug. Regarding statistics, present Serpent calculations showed an average deviation of $\pm 1.4 \mathrm{pcm}$ and Ref. [7,8] incorporated $\pm 2.0 \mathrm{pcm}$ uncertainty. Available standard deviation values $( \pm \sigma)$ of the benchmark cases are included in Table 2 below.

Table 2: 3D Full-Core Results for CA/OX Cores.

\begin{tabular}{|c|c|c|c|c|c|c|}
\hline Reference & $k_{\text {eff }}(-)$ & $K_{\mathrm{D}}(\mathrm{pcm})$ & $\mathrm{SVR}(\mathrm{pcm})$ & $\mathrm{CRW}(\mathrm{pcm})$ & $\Delta \rho_{\text {ax }}(\mathrm{pcm})$ & $\Delta \rho_{\mathrm{rad}}(\mathrm{pcm})$ \\
\hline$[6]$ & $1.0136 / 1.0096$ & $-1002 /-895$ & $2048 / 1932$ & $-4326 /-6092$ & - & - \\
\hline$\pm \sigma[6]$ & $0.0090 / 0.0048$ & $167 / 89$ & $398 / 171$ & $1124 / 995$ & - & - \\
\hline Present model & $1.0067 / 1.0120$ & $-945 /-936$ & $1552 / 1336$ & $-4217 /-6127$ & $-179 /-103$ & $-421 /-405$ \\
\hline$[7,8](\mathrm{OX})$ & 1.0107 & -852 & 1864 & -6046 & -120 & -429 \\
\hline
\end{tabular}

\section{FEW-GROUP CONSTANTS' GENERATION FOR ANTS}

\subsection{D Models and Boundary Conditions}

Supercell and colorset lattices were composed in order to obtain 2D homogenized few-group cross sections of non-fissile and fissile cells. In cases of interfaces between fissile and non-fissile cells colorset models were written for subchannels e.g. outer core FA with one, two or three adjacent radial reflector cells. Fuel lattices had periodic boundary conditions while other models e.g. control rod models were treated with reflective boundary conditions.
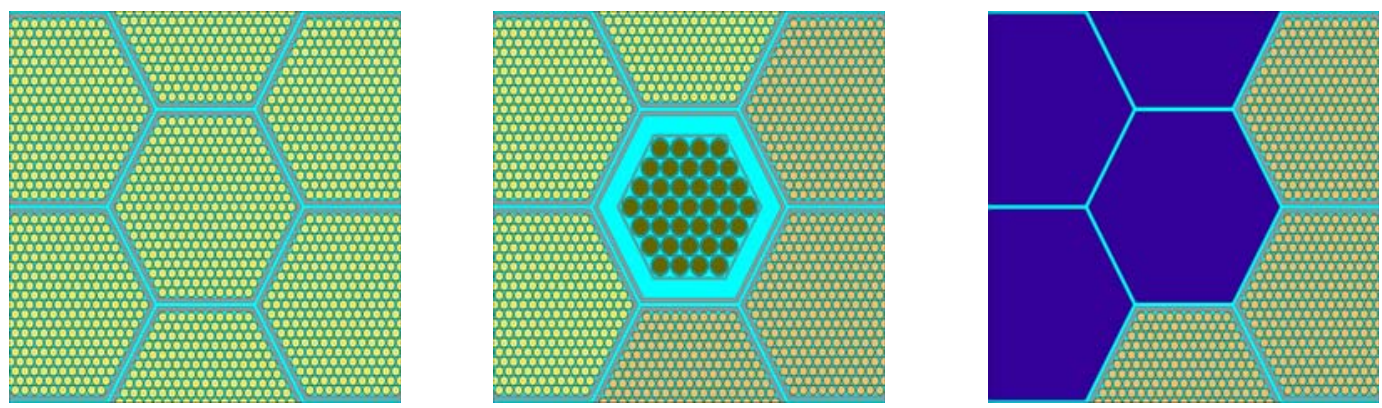

Figure 2: Serpent 2D Inputs (left - inner core infinite lattice, middle - primary CR, right radial reflector)

Regarding the few-group energy condensation the ECCO-33 structure was considered first, afterwards a simplified 24-group equal-lethargy bin structure was derived lumping the thermal region 
groups into one group (24th) hence reducing statistical errors of the lowest energy groups [9]. The SCALE238 structure was used for micro-group condensation.

A sensitivity study of homogenization methods was carried out as well, considering $B_{1}$ and $P_{1}$ leakage corrections and FM leakage correction with out-scattering diffusion coefficients. The study utilized a CA inner fuel 2D infinite lattice model in order to demonstrate the bias of the methods (see Fig. 2 left). Each run had 2500 cycles (2 000 active / 500 passive) with a population of 60000 neutrons. Calculations were performed with varying neutron populations between $2000-100000$ whereas it was concluded that statistical errors did not show noticeable variance with populations over 60000 particles, later on the same boundary conditions were applied in XS generation for Ants.

\subsection{Few-Group Cross Section Generation Sensitivity Study}

Group constants e.g. $\Sigma_{\mathrm{a}}, \nu \Sigma_{\mathrm{a}}, \kappa \Sigma_{\mathrm{f}}$ and $D$ describe materials in Ants, Fig. 3 represents the trends of the mentioned terms on the $10^{-11}-20 \mathrm{MeV}$ spectrum. It can be seen that for most cases the various methods showed little to negligible discrepancies except for the 24th energy-group where the $B_{1}$ leakage correction had the lowest XSs systematically. Regarding $\Sigma_{\mathrm{a}}, \nu \Sigma_{\mathrm{f}}, \kappa \Sigma_{\mathrm{f}}$ the average and maximum difference was $-0.24 / 0.64 \%$ between no-leakage and corrected values, respectively. As for the diffusion coefficient the maximum average difference was $1.52 \%$ given by the $P_{1}$ method followed by $B_{1}(0.33 \%)$ and out-scattering $(0.07 \%)$ that were noticeably lower than the $P_{1}$ error.

These tendencies confirm an earlier conclusion of Faure et al. [10] whereas leakage-corrected XS generation methods were studied in in fast systems. It was reported that the leakage models had a low impact on cross section generation. Such insensitivity can be explained by the fact that the introduction of a leakage term does not change noticeably the structure of the local multi-group flux within a macro-group. Considering scattering cross sections, in general for the scattering matrix the maximum error between leakage-corrected and no-leakage methods was $-0.23 \%\left(P_{1}\right)$.

\section{ANTS REDUCED-ORDER NODAL DIFFUSION SOLVER}

\subsection{Code and Input Structure}

The development of the Ants reduced-order nodal diffusion solver has started at VTT in 2018 $[11,12]$. The in-house built code is based on the analytic function expansion nodal (AFEN) - and function expansion nodal methods (FENM) $[13,14]$. The algorithm tackles the large number of nodal variables without transverse integration which is beneficial considering hexagonal geometry but has a negative impact on solver speed. The expansion of the intranodal flux is based on analytic basis functions which' solutions are obtained from the steady-state multi-group diffusion equation. Regarding the model structure the user has to provide basic geometry data e.g. core lattice pitch, number and length of axial nodes with respect to benchmark data [1]. The material of each node is described by a set of cross sections in a 24-group condensed format, extracted from the 2D supercell results as mentioned above. Finally convergence limits can be defined for $k_{\text {eff }}$ and fission source for instance, calculations presented in Section 4.2 had a $\varepsilon_{\mathrm{k}_{\mathrm{eff}}}=10^{-8}$ and $\varepsilon_{\mathrm{S}}=10^{-6}$ limits. 


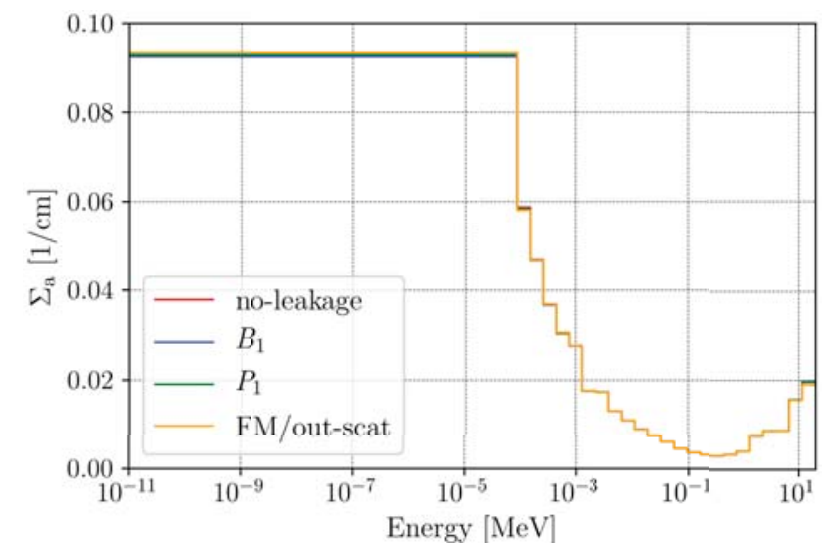

(a) $\Sigma_{\mathrm{a}}$

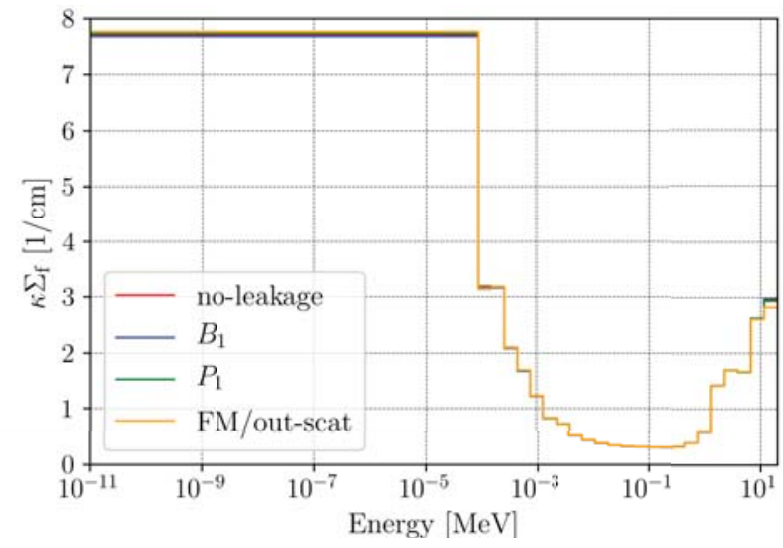

(c) $\kappa \Sigma_{\mathrm{f}}$

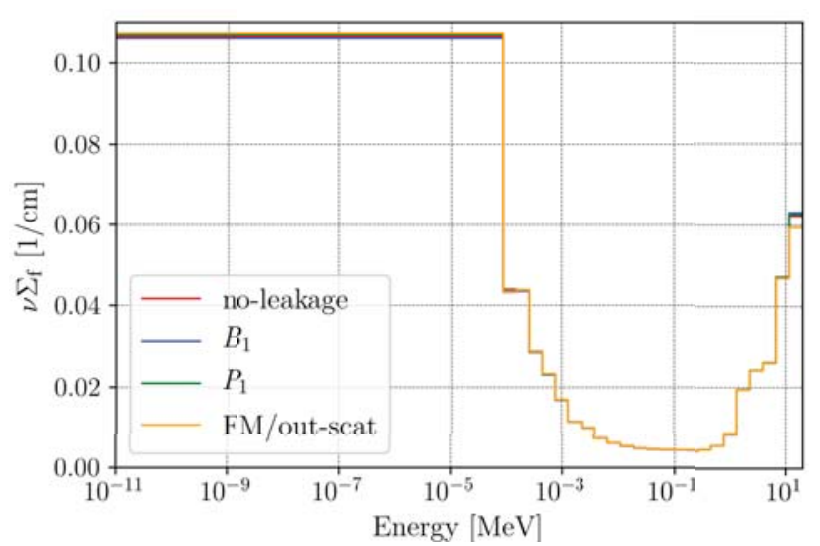

(b) $\nu \Sigma_{\mathrm{f}}$

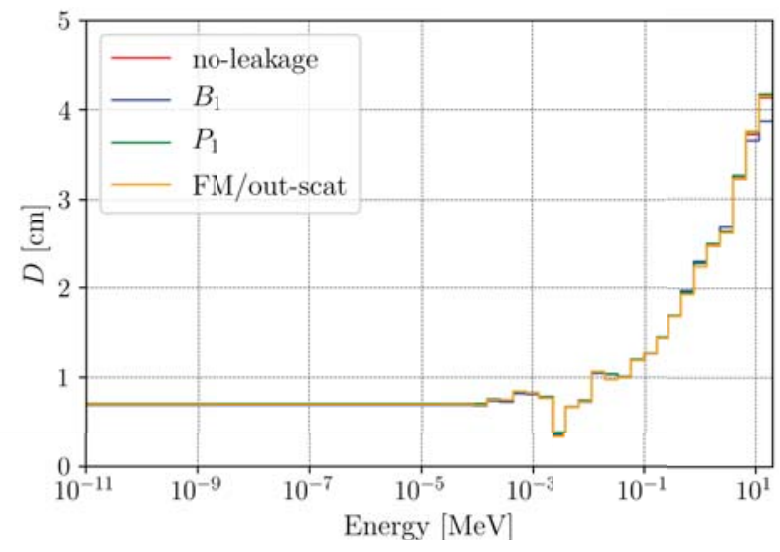

(d) $D$

Figure 3: Group Constants in CA Inner Fuel 2D Supercell.

\subsection{Comparison of Ants and Serpent}

In order to compare power distribution of Ants and Serpent cores the assembly-wise relative powers were normalized to the maximum value in each solution, the differences between these normalized distributions are depicted in Fig. 4. Comparing Serpent to Ants the average and absolute maximum error was $0.00 / 1.52 \%$ in case of CA and $0.19 / 2.66 \%$ for OX, respectively.

The axial power distribution comparison is shown in Fig. 5 highlighting that Ants provided a more symmetric distribution for CA than Serpent meaning that the CRs had smaller worth in the reduced-order model as discrepancies started to increase in the upper section of the core. The OX core followed the axial profile of Serpent better in the upper part, noticeable $+0.39 \%$ and $+1.85 \%$ for CA and $+0.52 \%$ and $+2.23 \%$ for OX, respectively.

The Ants radial power profiles (see Fig. 6) show good agreement with the MC results with largest absolute error of $1.26 \%$ for CA and $1.86 \%$ for OX, comparing Ants to MC. The label "Ants-simp." denotes a simplified case where the local geometry was neglected on the radial reflector/outer core boundary i.e. outer core FA 2D infinite lattice cross sections were used for fuel assemblies with 


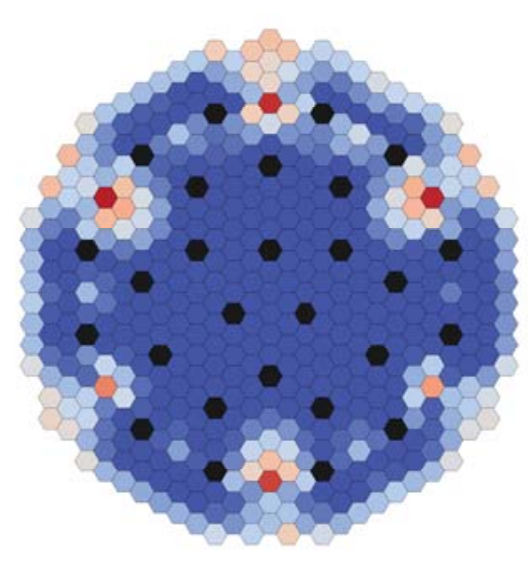

(a) $\mathrm{CA}$
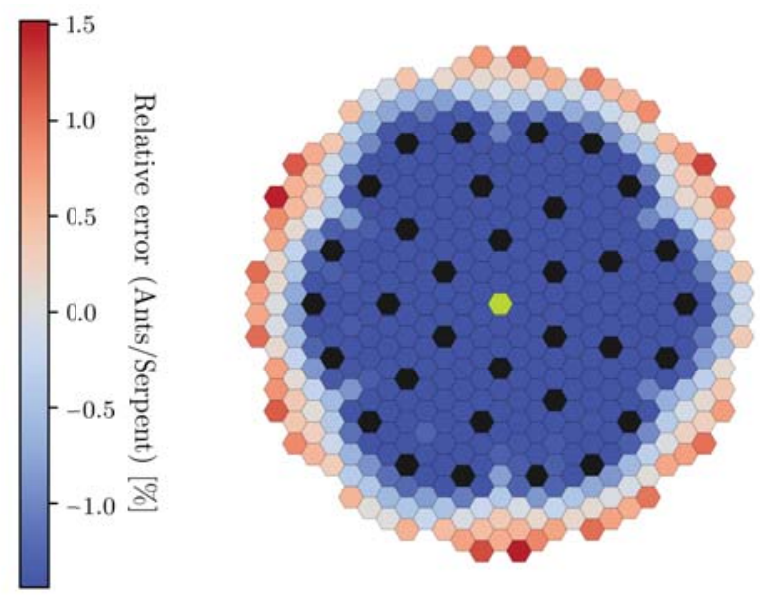

(b) $\mathrm{OX}$

Figure 4: Comparison of Power Distributions in Criticality Case.

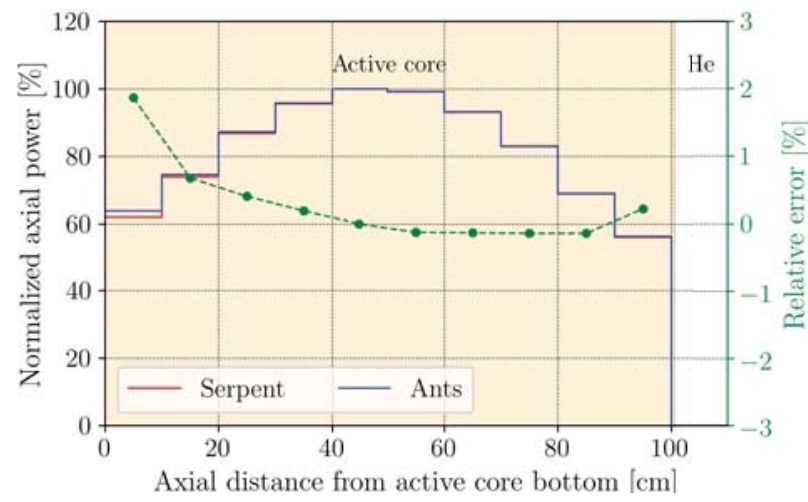

(a) $\mathrm{CA}$

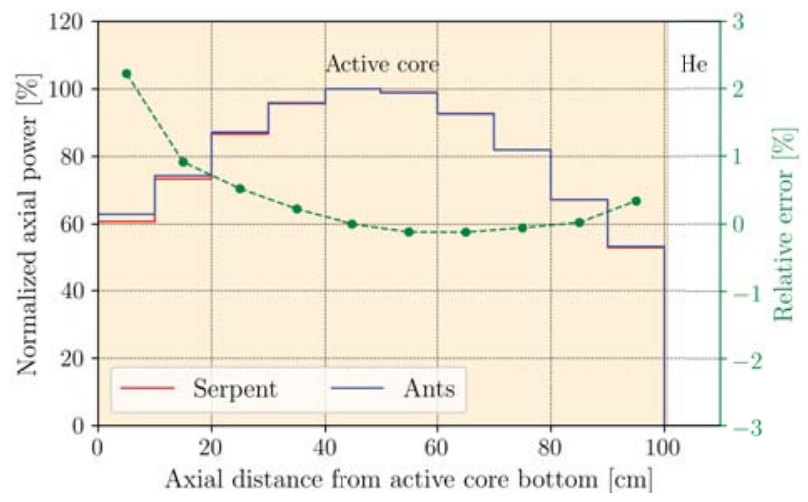

(b) OX

Figure 5: Axial Power Profiles in Criticality Case.

reflector neighbours. The deviation is clear between the two CA Ants results indicating that local heterogeneities affecting flux did alter the global results, moreover, the maximum absolute error increased to $2.17 \%$. The OX results are similar comparing maximum absolute errors where the finer mesh and the simplified case gave $1.87 \%$ and $3.03 \%$, respectively. Hence the OX system with larger flux gradient on the outer core edge was more sensitive to perturbation from mesh resolution.

Table 3 summarizes the Ants-Serpent comparisons considering several test cases described in Section 2.3. Regarding the criticality case the discrepancies between $k_{\text {eff }}$ results were $7 \mathrm{pcm}(\mathrm{CA})$ and $136 \mathrm{pcm}(\mathrm{OX})$. Even though the OX error is significantly larger than its CA counterpart, earlier studies on the OX core showed similar discrepancy ranges with Serpent-PARCS (84 pcm) and Serpent-DYN3D $(128 \mathrm{pcm})$ [7]. Has to be mentioned that using superhomogenization (SPH) the 


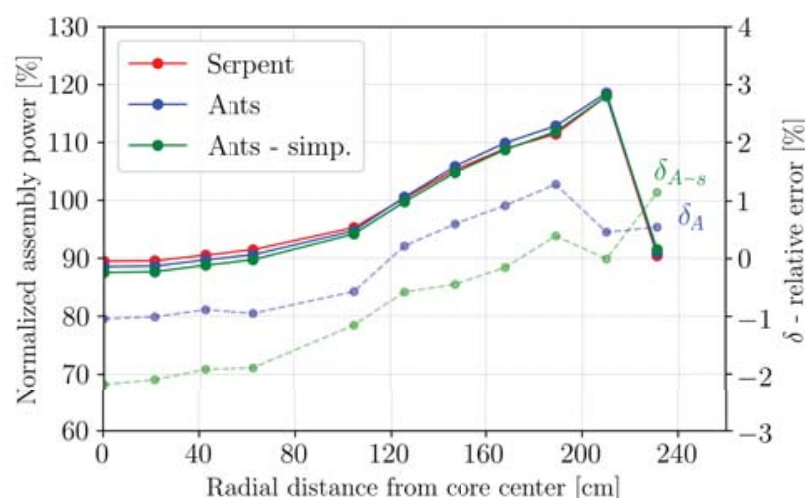

(a) $\mathrm{CA}$

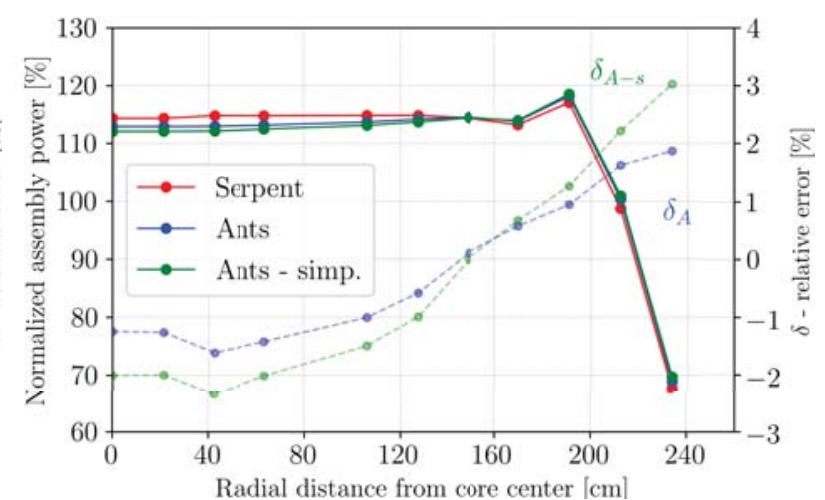

(b) $\mathrm{OX}$

Figure 6: Radial Power Profiles (in Main Diagonal.

nodal solution can be significantly improved as reported in [15]. SVR coefficients show larger departure comparing Ants to Serpent, further analysis is required to study the effect of self-shielding in heterogeneous and homogeneous codes. Looking at CRW, $\Delta \rho_{\text {ax }}$ and $\Delta \rho_{\text {rad }}$ the trends are acceptable in general although $\Delta \rho_{\text {ax }}$, Ox did not show satisfying similarity between the high fidelity and the reduced-order values. This underlines that the depiction (by 2D homogenization) of a composition-perturbation in fuel material can translate into varying discrepancies comparing stochastic and deterministic codes.

Table 3: 3D Full-Core Results for CA/OX with High-Fidelity and Reduced-Order Solvers.

\begin{tabular}{|c|c|c|c|c|c|c|}
\hline Code & $k_{\text {eff }}(-)$ & $K_{\mathrm{D}}(\mathrm{pcm})$ & $\mathrm{SVR}(\mathrm{pcm})$ & $\mathrm{CRW}(\mathrm{pcm})$ & $\Delta \rho_{\mathrm{ax}}(\mathrm{pcm})$ & $\Delta \rho_{\text {rad }}(\mathrm{pcm})$ \\
\hline Serpent & $1.00670 / 1.01198$ & $-945 /-936$ & $1552 / 1336$ & $4217 / 6127$ & $-179 /-106$ & $-421 /-405$ \\
\hline Ants & $1.00806 / 1.01241$ & $-947 /-957$ & $1895 / 1290$ & $4212 / 6180$ & $-182 /-159$ & $-414 /-404$ \\
\hline
\end{tabular}

\section{CONCLUSIONS}

The Serpent full-core 3D simulation of two SFR cores was presented and compared to benchmark data [6] where Serpent showed satisfying agreement with previous works considering heterogeneous geometry description. The 2D supercell models highlighted the insensitivity of few-group constants to leakage-correction methods in a fast system. The methodology and performance of the Ants reduced-order diffusion solver was demonstrated as a potential surrogate of Serpent. Reactivity feedbacks showed good agreement with benchmark data and MC results however larger differences were observed in cases of SVRs and axial fuel expansion of the OX core indicating that the applied homogenization had larger impact on composition-related perturbations compared 
to changes in geometry. Further studies are needed to improve the efficiency of group constant generation with special care to fissile and non-fissile cell interfaces.

\section{References}

[1] D. Blanchet, L. Buiron, N. Stauff, T. K. Kim, T. Taiwo, AEN - WPRS Sodium Fast Reactor Core Definitions (v1.2), OECD/NEA, 19th September 2011.

[2] J. Leppänen, M. Pusa, T. Viitanen, V. Valtavirta, T. Kaltiaisenaho, "The Serpent Monte Carlo code: Satus, development and applications in 2013" Annals of Nuclear Energy, Volume 82, pp. 142-150 (2015).

[3] Terje Tverberg, Mixed-oxide (MOX) fuel performance benchmark, Summary of the Results for the Halden Reactor Project MOX Rods, NEA/NSC/DOC(2007)6, OECD HRP, 2007.

[4] П. Л. Кириллов, Теплофизические свойства материалов ядерной техники - учебное справочное пособие 2-е изд., Обнинский Институт Атомной Энергетики, Обнинск, Russian Federation (2007).

[5] Ю. Г. Годин, А. В. Тенишев, Карбидное ядерное топливо, Московский ИнженерноФизический Институт, Москва, Russian Federation (2007).

[6] L. Buiron et al., "Evaluation of Large 3600 MWth Sodium-Cooled Fast Reactor Neutronic OECD benchmarks" Proceedings of PHYSOR 2014, Kyoto, Japan, September 28 - October 3, (2014).

[7] E. Nikitin, E. Fridman, K. Mikityuk, "Solution of the OECD/NEA neutronic SFR benchmark with Serpent-DYN3D and Serpent-Parcs code systems" Annals of Nuclear Energy, Volume 75, pp. 492-497 (2014).

[8] E. Nikitin, E. Fridman, "Extension of the reactor dynamics code DYN3D to SFR applications - Part I: Thermal expansion models" Annals of Nuclear Energy, Volume 119, pp. 382-389 (2018).

[9] E. Fridman, "Generation of few-group constants with Serpent: Application examples" Proceedings of PHYSOR 2014, Kyoto, Japan, September 28 - October 3, (2014).

[10] B. Faure, G. Marleau, "Simulation of a sodium fast core: Effect of $B_{1}$ leakage models on group constant generation" Annals of Nuclear Energy, Volume 99, pp. 484-494 (2017).

[11] V. Sahlberg, A. Rintala, "Development and first results of a new rectangular nodal diffusion solver of Ants" Proceedings of PHYSOR 2018, Cancún, Mexico, 22-26 April, (2018).

[12] A. Rintala, V. Sahlberg, "Extension of nodal diffusion solver of Ants to hexagonal geometry" Kerntechnik, Volume 84, pp. 252-261 (2019).

[13] N. Z. Cho, J. M. Noh, "Analytic function expansion nodal method for hexagonal geometry" Nuclear Science and Engineering, Volume 121, pp. 245-253 (2014).

[14] B. Xia, Z. Xie, "Flux expansion nodal method for solving multigroup neutron diffusion equations in hexagonal-z geometry" Annals of Nuclear Energy, Volume 33, pp. 370-376 (2006).

[15] E. Nikitin, E. Fridman, K. Mikityuk, "On the use of the SPH method in nodal diffusion analyses of SFR cores" Annals of Nuclear Energy, Volume 85, pp. 544-551 (2015). 